\title{
HAND-ASSISTED LAPAROSCOPIC NEPHRECTOMY IN LIVING DONOR
}

\author{
LUIZ S. SANTOS, ANDRÉ E. VARASCHIN, FERNANDO MEYER, ALCIDES BRANCO, \\ FERNANDO KOLESKI, RONALDO CARVALHO \\ Division of Urology, Unit of Renal Transplantation, Cajuru University Hospital, Catholic University of \\ Paraná (PUC - PR), Curitiba, Paraná, Brazil
}

\begin{abstract}
Objective: Report the authors' initial experience with hand-assisted laparoscopic nephrectomy technique in renal donors for transplantation.

Materials and Methods: Twenty-seven donors submitted to hand-assisted laparoscopic nephrectomy were retrospectively analyzed from February 2001 to June 2002. Technical aspects of the donor surgery, results, and complications, are discussed, as well as recipient's complications and outcomes.

Results: Among 27 hand-assisted laparoscopic nephrectomies, left kidney was withdrew in 18 donors (66.6\%), and right kidney in 9 (33.3\%). The operative time ranged from 55 to 210 minutes (mean $132.7 \pm 37.6 \mathrm{~min}$ ), and the time of hot ischemia ranged from 2 to 11 minutes (mean $4.7 \pm 2.5$ $\mathrm{min}$ ), with an estimated mean blood loss during the intraoperative period of $133.3 \mathrm{~mL}$. Conversion to open surgery was necessary for $1(3.7 \%)$ patient due to vascular lesion. In graft evaluation, immediate diuresis was observed in 26 (96.3\%) cases, and mean serum creatinine in PO day 7 was $1.5 \pm 1.1 \mathrm{mg} /$ dL. Renal vein thrombosis occurred in $1(3.7 \%)$ patient requiring graft removal. Lymphocele was observed in 3 recipients (11.1\%), and urinary leakage due to ureteral necrosis in 1 case (3.7\%).

Conclusion: Hand-assisted laparoscopic nephrectomy in living donors is a safe procedure and an effective alternative to open nephrectomy. In this series, the procedure presented low morbidity after surgery providing to the recipient a good morphological and functional quality of the graft.
\end{abstract}

Key words: kidney; kidney transplantation; laparoscopy; living donors

Int Braz J Urol. 2003; 29: 11-7

\section{INTRODUCTION}

Minimally invasive nephrectomy in a living donor is described as a surgical modality that uses the classic laparoscopic technique combined or not to the application of the surgeon hand as a support tool during the renal dissection maneuver. Hand-assisted techniques use abdominal wall incision - required for removing the organ in its integrity —, as an access to the kidney, generally employing devices that seal the incision, avoiding thus the loss of the pneumoperitoneum gas. Hand-assisted laparoscopic nephrectomy (HLN) provides the performance of the procedure under an excellent laparoscopic visualiza- tion in an enlarged operative field, which is substantially facilitated by hand support (1). We describe here our initial experience with 27 donors submitted to HLN, exhibiting technical data of the procedure, its morbidity, its complications, as well as ease and difficulties observed in this approach.

\section{MATERIALS AND METHODS}

Medical data of 27 consecutive HLN performed in renal donors from February 2001 to June 2002 in our unit were retrospectively analyzed. Donor demographic characteristics, such as sex, age, and 
some parameters, such as hospital stay, estimated blood loss, surgical time, hot ischemy time, and intra- and post-operative complications were analyzed. Graft function was evaluated for immediate presence of diuresis after renal reperfusion and for serum creatinine on PO day 7. Vascular and ureteral complications of the recipient were also observed.

All donation candidates were submitted to preoperative routine evaluation. The requested exams were those usually performed for conventional renal donor, including digital angiography and intravenous pyelogram for the renal vascular anatomy and colleting system study. Left kidney nephrectomy was preferably performed, since the left kidney has a longer renal vein, which significantly facilitates the graft, excluding those cases where its preservation had a clear benefit for the donor.

The surgical procedure is initiated with the patient positioned in partial lateral decubitus $\left(30^{\circ}\right)$, contralateral to the kidney to be removed, under general anesthesia and continuous peridural blockade. The delimitation of the area for the device application allowing assisted laparoscopy is performed, as well as the trocar disposition as illustrated in Figure1. Transperitoneal access was performed in all cases. The extension of the skin incision must be of the same size in centimeters of the glove used by the assistant, approximately 7 to $8 \mathrm{~cm}$. The incision is initiated in the lateral border of the rectus abdominis muscle, ex-

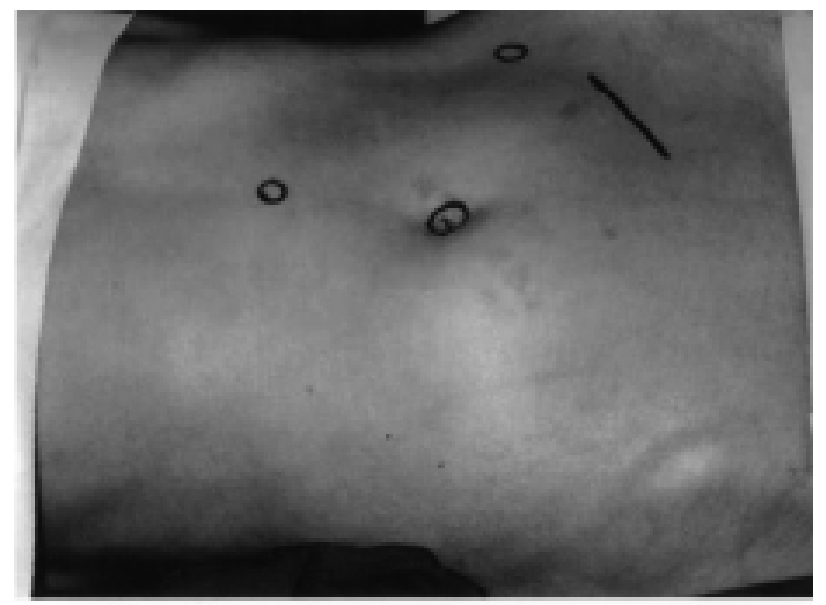

Figure 1 - Ports dispositions and localization of the incision for left nephrectomy.

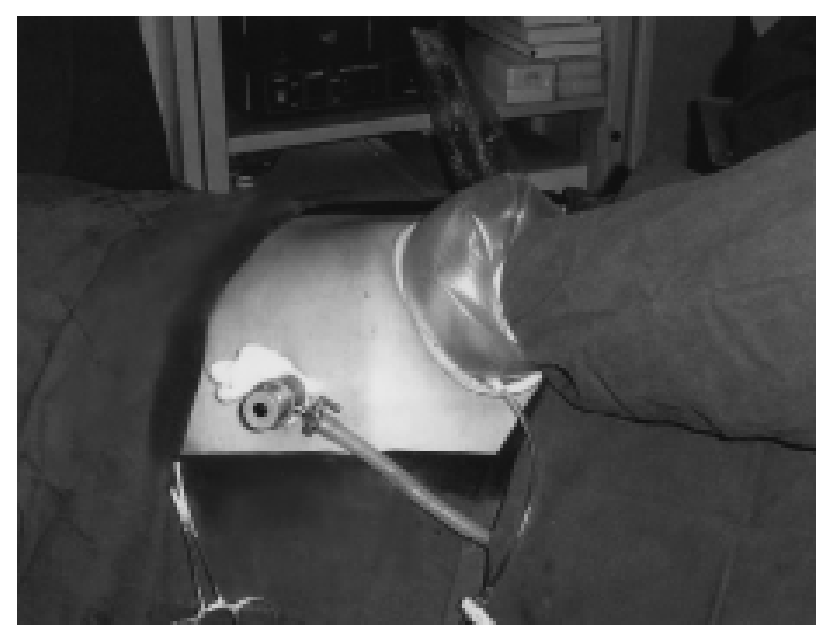

Figure 2 - Device installed for hand-assisted laparoscopy.

tending in an oblique fashion towards the spina iliaca anterior superior. The obliquus externus and internus abdominis muscles, and the transversalis abdominis muscle are divulsed, the peritoneum opened and the colon medially mobilized. The ureter is identified, isolated with a Penrose drain, and dissected in superior and inferior direction up to the crossing of the iliac vessels. Only then, the first $10 \mathrm{~mm}$ trocar (camera) is inserted hand-assisted in the umbilicus. The device (hand-port) is installed according to the manufacturer recommendations, and the assistant's hand is inserted in the abdominal cavity (Figure-2). The peritoneum is insufflated with $\mathrm{CO}_{2}$, submitted to a pressure of $15 \mathrm{~mm} \mathrm{Hg}$, and secondary openings are created under laparoscopic control. A trocar of $5 \mathrm{~mm}$ is placed at the level of the midclavicular line, about $3 \mathrm{~cm}$ below the left costal border for the dissectors forceps, and another of $12 \mathrm{~mm}$ trocar is inserted at the level of the anterior axillary line above the crista iliaca for the insertion of the scissors and the vascular stapler. A third accessory port can be established, when necessary, at the level of the middle axillary line, to assist in presenting the kidney or to cavity aspiration.

In this series, 3 devices commercially available were used to perform the procedure. In the initial 9 cases, we used Intromit ${ }^{\circledR}$ (Medtech Ltd, Clara, Ireland Rep.) (Figure-2). The Omniport ${ }^{\circledR}$ (Weck Closure Systems, Charlote, NC, USA) was used in 16 
patients, and in the last 2 cases, the Lap Disc ${ }^{\circledR}$ (Ethicon Endo-Surgery, Cincinnati, OH, USA) was installed. All equipments had an easy installation providing comfort and freedom to the surgeon in the performance of the maneuvers required during surgery.

After the hand and trocar insertions, and the inspection of the cavity, kidney dissection is initiated according to the technique described by Nakada (2). In all the procedures the harmonic scalp - UltraCision ${ }^{\circledR}$ (Ethicon Endo-Surgery, Cincinnati, OH, USA) was used for the dissection and coagulation of vessels and perirenal structures. Once completed the kidney, ureter and renal pedicle dissections, the renal artery clamping is performed with 3 proximal metallic clips and one distal metallic clip and its immediate section is performed. The distal artery ligature was used in the first 5 cases and eliminated afterwards to better preserve a greater length of the vessel. The renal vein is then tied and sectioned with a linear vascular stapler - Endo-GIA ${ }^{\circledR} 45 \mathrm{~mm} / 2.5 \mathrm{~mm}$ (U.S. Surgical Corp., Norwalk, USA), and the kidney is removed through the port, performing at last the ligature and distal section of the ureter at the level of the iliac vessels crossing. In some occasions, the renal vein ligature was performed with cotton suture 2-0 and 2 metallic clips over the knot, afterwards sectioning the vein with scissors, without significant decrease in its length. In this situation, the suture is passed through the vein before renal artery ligature, leaving the loose knot to be tightened only after sectioning the artery. Hemostatic revision is performed under direct vision, and a Penrose drain is inserted in the abdominal cavity and removed after 24 hours. At last, the synthesis of the muscle wall and skin is performed.

The surgical approach for the right kidney used in our Unit is the same for the left kidney, that is, through an oblique inguinal incision using, however, the assistant's left hand as a tool for internal dissection and, frequently, a subcostal portal to retract the liver.

\section{RESULTS}

Table-1 shows demographic data and surgical results observed in 27 donors submitted to HLN.
Table 1 - Hand-assisted laparoscopic nephrectomy results.
$\mathrm{N}$ of patients

$\operatorname{Sex}(\mathrm{M} / \mathrm{F})$

Mean age (years)

Mean surgical time (min)

Mean hot ischemy time ( $\mathrm{min}$ )

Mean hospital stay (days)
Nephrectomy side (L/R) (\%)

\section{7}

$13 / 14$

$18 / 966.6 / 33.3 \%$

$38.8(22-62)$

$132.7(55-210)$

$4.7(2-11)$

$3.8(3-7)$
Among these, 13 were males, and 14 females, with age ranging from 22 to 62 (mean 38.8 years) years. Laparoscopic nephrectomy was left in 18 cases, and right in 9 cases.

Surgical time ranged from 55 to 210 minutes (mean $132.7 \pm 37.6 \mathrm{~min}$ ), this parameter defined as the time passed from the moment of the incision until the skin synthesis finalization. Estimated blood loss was of 30 to $600 \mathrm{~mL}$ (mean $133.3 \mathrm{~mL}$ ), and no patient needed transfusion. The time of hot ischemy ranged from 2 to 11 minutes (mean $4.7 \pm 2.5 \mathrm{~min}$ ), related in some situations to the vascular complexity verified in 3 patients $(11.1 \%)$ with renal artery duplication. Mean time hospital stay for the donor was of 3.8 days, ranging from 3 to 7 days.

All the patients submitted to HLN concluded the procedure as planned, that is, with the intact removal of the organ, with vascular and ureteral preservation adequate to the graft, excluding one patient whose procedure was complicated by the accidental lesion of the left adrenal vein during the dissection maneuvers, leading to moderate bleeding and needing to convert to open surgery. In this case, an anterior subcostal transverse incision was performed and the nephrectomy concluded with a good outcome for the patient and the graft.

Intraoperative complications of HLN for the donor included vascular lesions in 3 cases $(11.1 \%)$, of which laceration of renal artery (1 case), of the gonadal vein (1 case), and of the adrenal vein (1 case), the latter presenting moderate bleeding, requiring conversion to open surgery. The first 2 lesions mentioned were small and corrected laparoscopically with metallic clips. Transfusion was not necessary in any case. Postoperative complications in donors are listed 
Table 2 - Postoperative complications in renal donors submitted to hand-assisted laparoscopic nephrectomy.

\begin{tabular}{lcl}
\hline Complications & No. of Patients (\%) \\
\hline Ileum & 2 & $(7.4)$ \\
Urinary infection & 2 & $(7.4)$ \\
Pneumonia & 1 & $(3.7)$ \\
Transfusion & 0 & $(0)$ \\
Mortality & 0 & $(0)$ \\
Total & 5 & $(18.5)$ \\
\hline
\end{tabular}

in Table-2, and corresponded to $18.5 \%$ of the cases. Urinary infection occurred in 2 patients, and pneumonia in 1, all treated with adequate antibiotic therapy and complete resolution of the infection. No cases of surgical wound infection were observed, and no death occurred in consequence of the procedure. The major complication observed was persistent vomit during between 2 to 6 days in 6 patients $(22.2 \%)$, possibly due to peridural anesthetic blockade with morphine, used in the beginning of this series, associated with intestinal loops manipulation. Afterwards, the peridural anesthesia was abolished, with significative decrease in the postoperative vomits in the last 12 cases.

Vascular and ureteral complications rates for the recipient are demonstrated in Table-3, together with renal graft evaluation data. Immediate diuresis after renal reperfusion was observed in $96.3 \%$ of the cases. Serum creatinine observed in the PO day 7 ranged from 0.8 to $6.4 \mathrm{mg} / \mathrm{dL}$ (mean $1.5 \pm 1.1 \mathrm{mg}$ / $\mathrm{dL}$ ), with all the patients presenting satisfactory diuresis and creatinine decline in this period, except one patient that progressed with anuria due to renal vein thrombosis, needing graft removal and consequent hemodialysis. Lymphocele occurred in 3 recipi-

Table 3 - Recipient evolution results.

\begin{tabular}{lcc}
\hline Mean blood creatinine -7 PO (mg/dL) & 1.5 & $(0.8-6.4)$ \\
Immediate diuresis (\# cases) & 26 & $(96.3 \%)$ \\
Lymphocele & 3 & $(11.1 \%)$ \\
Urinary fistula & 1 & $(3.7 \%)$ \\
Renal vein thrombosis & 1 & $(3.7 \%)$ \\
\hline
\end{tabular}

ents, and 2 cases were medically conducted due to its small volume. One patient required lymphocele laparoscopic treatment with good outcomes. One patient presented urinary fistula and renal function impairment in PO day 6 due to terminal ureteral necrosis, needing surgical intervention with reimplant and placement of an ureteral catheter. There was resolution of the fistula and normalization of the graft function.

\section{DISCUSSION}

Renal transplant with living donor is admittedly superior to transplant with cadaveric kidney, especially for the survival of the patient and the graft (3). The known technical, legal, and cultural difficulties for the organ procurement in our context make the living donor the major hope for obtainment of a kidney to transplantation. Actually, the search for technical improvement intending to sooth the aggression suffered by the donor is constant. HLN seems today to be the best adapted method to this context, combining the advantages of a minimally invasive surgery to the security and easiness of manual dissection, decreasing the learning curve and reducing intraoperative complications (3). In instances where the organ should be removed intact through an incision, as in the nephrectomy with living donor, or radical nephrectomy due to a tumor, the surgery handassisted seems to offer a significative benefice (1).

In the last 2 decades, there has been a growing interest in minimally invasive surgeries with less morbidity as laparoscopy. In 1990, Clayman et al. described the first laparoscopic nephrectomy performed in the treatment of a renal tumor (4), and ever since many institutions worldwide used this technique in the treatment of malignant and benign renal diseases $(5,6)$. In 1995, Ratner et al. reported the first successful laparoscopic nephrectomy with living donor, the kidney being removed through a $9 \mathrm{~cm}$ infraumbilical median incision (7). Later, Wolf et al performed the first hand-assisted laparoscopic nephrectomy with living donor (1). This procedure has obtained great acceptance in many transplant centers, and new devices have emerged in the market with 
intending to ease and simplify each time more this new and fascinating surgical technique $(2,8)$.

Several reports confirmed the advantages of hand-assisted laparoscopic nephrectomy compared to open surgery for the donor, establishing that it may be performed with safely and without harm for the donor, and the graft function $(9,10)$. Surgical time is comparable to open nephrectomy, and tends to decrease with the learning curve, as established by Wolf et al., who obtained a decrease from 252 to 85 minutes in the presented series (1). In this study, mean surgery time was 132.7 minutes, slightly bellow the mean observed by other authors $(11,12)$. Some attitudes were important for the reduction of surgical time observed in this series. The oblique inguinal incision for the glove insertion was initially used to perform colon mobilization and ureter dissection under direct vision as far as the incision permitted. Also, using the assistant's hand for the internal dissection maneuvers, allowing the surgeon to work freely with 2 ports. These technical changes optimized the surgery and decrease its length.

HLN permits a safe renal dissection in an ample operative field, promoting an excellent renal pedicle exposition. Jacobs et al. presented a conversion rate to open surgery of $1.6 \%$ (13). In this series, only one conversion $(3.7 \%)$ was necessary, due to vascular lesion. Mean hot ischemy time was of 4.7 minutes, being superior to Ruiz-Deya et al. (12), and Wolf et al. (11) experiences', that reported 1.8 and 2,9 minutes, respectively. Yet, this time did not result in harm to the graft function as observed in the great majority $(96.3 \%)$ of the recipients, that presented immediate diuresis and mean serum creatinine of 1.5 $\mathrm{mg} / \mathrm{dL}$. Some studies comparing HLN with classic laparoscopic nephrectomy observed that HLN decreases the surgery and hot ischemy time, in addition to promoting better safety in its performance $(10,12)$.

The incidence of ureteral complications after laparoscopic nephrectomy in the recipient may vary from 2 to $11 \%$ according to literature reports $(3,14)$. In this series, $1(3,7 \%)$ patient developed urinary fistula secondary to a terminal ureteral necrosis, which lead us to more caution in ureter dissection, seeking to preserve more periureteral fat. Renal vein thrombosis, which leads to the graft removal, was possibly a consequence of the technical difficulty observed in right side nephrectomy, resulting in shorter vessels, that made difficult the anastomosis. Right HLN is frequently more difficult due to the necessity to move displace the liver and, in addition, the renal vein length can be a problem in the recipient implant. Advanced age and obesity are not considered contraindications to the HLN, neither is renal vessel multiplicity (15). In this study, we found renal artery duplicity in 3 patients whose graft outcome was similar to those with only one artery.

Laparoscopic nephrectomy with living donor is an emerging technique that has not gained yet wide acceptation in the community involved in transplants, possibly due to the technical difficulties it presents. However, the advantages related to decrease in donor morbidity, less time of hospital stay, better healing, and excellent graft functional quality for the recipient, turn this technique very attractive, and may greatly increase the number of donors (13).

\section{CONCLUSION}

Hand-assisted laparoscopic nephrectomy with living donor associates low from the laparoscopic methods to the intra-abdominal organs manipulation provided by the surgeon's hand. In this study, the results obtained with this method are comparable to data published in literature. Limited discomfort, as well as low morbidity observed in this series, to the donor as well as to the recipient, make this technique very attractive.

\section{REFERENCES}

1. Wolf JS Jr, Tchetgen MB, Merion RM: Hand-assisted laparoscopic living donor nephrectomy. Urology. 1998; 52: 885-7.

2. Nakada SY: Techniques in endourology. Hand-assisted laparoscopic nephrectomy. J Endourol. 1999; 13: 915.

3. Brown SL, Biehl TR, Rawlins MC, Hefty TR: Laparoscopic living donor nephrectomy: a comparison with the conventional open approach. J Urol. 2001; 165: 766-9.

4. Clayman RV, Kavoussi LR, Soper N: Laparoscopic nephrectomy.initial case report. J Urol. 1991; 146: 278. 
5. Dunn MD, Portis AJ, Shalhav AL, Elbahnasy AM, Heidorn C, McDougall EM, et al.: Laparoscopic versus open nephrectomy: a 9-year experience. J Urol. 2000; 164: 1153-9.

6. Portis AJ, Elnady M, Clayman RV: Laparoscopic radical/total nephrectomy: a decade of progress. J Endourol. 2001; 15: 345-54.

7. Ratner LE, Ciseck LJ, Moore RG, Cigarroa FG, Kaufman HS, Kavoussi LR: Laparoscopic living donor nephrectomy. Transplantation. 1995; 60:1047-9.

8. Tokuda N, Nakamura M, Tanaka M, Naito S: Handassisted laparoscopic living donor 'nephrectomy using newly produced lap disc $®$ : initial three cases. J Endourol. 2001; 15: 571-4.

9. Stifelman MD, Hull D, Sosa E, SU LM, Hyman M, Stubenbord W, et al.: Hand assisted laparoscopic nephrectomy: a comparison with the open approach. J Urol. 2001; 166: 444-8.

10. Wolf JS Jr, Moon TD and Nakada SY: Hand assisted laparoscopic nephrectomy: comparison to standart laparoscopic nephrectomy. J Urol. 1998; 160: 22-7.
11. Wolf JS Jr, Marcovich R, Merion RM, Konnak JW: Prospective, case matched comparison of hand assisted laparoscopic and open surgical living donor nephrectomy. J Urol. 2000; 163: 1650-3.

12. Ruiz-Deya G, Cheng S, Palmer E, Thomas R, Slakey D: Open donor, laparoscopic donor and hand assisted laparoscopic donor nephrectomy: a comparison of outcomes. J Urol. 2001; 166: 1270-4.

13. Jacobs SC, Cho E, Dunkin BJ, Flowers JL, Schweitzer E, Cangro C, et al.: Laparoscopic living donor nephrectomy: the university of Maryland 3-year experience. J Urol. 2000; 164: 1494-9.

14. Chan DY, Fabrizio MD, Ratner LE, Kavoussi LR: Complications of laparoscopic living donor nephrectomy: the first 175 cases. Transpl Proc. 2000; 32: 778.

15. Tan HP, Maley WR, Kavoussi LR, Montgomery RA, Ratner LE: Laparoscopic living donor nephrectomy: evolution of a new standard. Curr Opin Organ Transplantation. 2000; 5: 312-8.

Received: August 30, 2002 Accepted after revision: January 7, 2003

\author{
$\overline{\text { Correspondence address: }}$ \\ Dr. Luiz Sergio Santos \\ Rua Visconde de Nacar, 865 / 507 \\ Curitiba, PR, 80410-201, Brazil \\ Fax: + $5541233-6412$ \\ E-mail: 1ss@mps.com.br
}

\section{EDITORIAL COMMENT}

Since the first laparoscopic nephrectomy performed by Clayman et al. (1) in 1990, at the Washington University School of Medicine, urologic laparoscopy has remarkably evolved and, already in 1994 at the same University, the first work related to living donor nephrectomy was performed (2). Today, this technique is widespread in the world, and now in Brazil there is a group experienced in this method, as reported, with 27 cases.
This group favored the hand-assisted technique, as do Velidedeoglu et al. (3), in a comparative analysis between pure laparoscopy and open surgery, which helps teaching this technique to the residents, providing extra safety to the donor, chiefly for less experienced surgeons. Nonetheless, Shalhav et al. (4) innovated with a pure laparoscopy technique with manual extraction without the device to hand-assisted technique, which, further than its great cost-effectiveness, 
also reduced significantly hot ischemia time. This latter method seems even more interesting for the underdeveloped countries that do not have disposable devices, which are costly and barely available.

Currently, major transplant centers perform laparoscopic nephrectomy in living donor, and this shall not be different in Brazil in a while. This technique, in addition to the benefits inherent to the minimally invasive method, presents a further advantage for the unit where it is performed, i.e., increasing the number of donations by living donors.

Alas, there are problems all over the world: at Washington University School of Medicine, where almost everything that exists in laparoscopy initiated, laparoscopic nephrectomy in living donor is not yet performed and, unfortunately, not for lacking surgeons experienced with the method.

\section{References}

1. Clayman RV, Kavoussi R, Soper N: Laparoscopic nephrectomy: initial case report. J Urol. 1991; 146:278.

2. Gill IS, Carbone JM, Clayman RV, Faddeb PA, Stone MA, Lucas BA, et al.: Laparoscopic live-donor nephrectomy. J Endourol. 1994; 8:143-8.

3. Velidedeoglu E, Williams N, Brayman KL, Desai NM, Campos L, Palanjian M, et al.: Comparison of open, laparoscopic, and hand-assisted approaches for live donor nephrectomy. Transplantation. 2002; 74:169-72.

4. Shalhav AL, Siqueira TM Jr, Gardner TA, Paterson RJ, Stevens LH: Manual specimen retrieval without a pneumoperitoneum preservation for laparoscopic live donor nephrectomy. J Urol 2002; 168:941-4.

5. Schweitzer EJ, Wilson J, Jacobs S, Machan CH, Philosophe B, Farney A, et al.: Increased rates of donation with laparoscopic donor nephrectomy. Ann Surg. 2000; 232:392-400.

Dr. Cassio Andreoni

Section of Endourology and Laparoscopy

Division of Urology, Federal University of São Paulo

São Paulo, SP. Brazil 